\title{
DESCRIÇÃO DA ÁREA DE ENFERMAGEM: Oferta e demanda dos cursos de graduação ${ }^{1}$
}

\author{
Maria Auxiliadora Córdova Christófaro ${ }^{2}$ \\ Maria da Glória Lima ${ }^{3}$ \\ Maria Jenny Silva Araujo ${ }^{4}$ \\ Maria Therezinha Nóbrega da Silva ${ }^{5}$
}

\section{INFORMAÇÕES PRELIMINARES}

Com a vigência da Lei $n^{\circ}$ 9131/95 e a conseqüente criação do Conselho Nacional de Educação (CNE), compete à SESu, entre outras atribuições, realizar estudos, preparar e instruir processos, elaborar relatórios e emitir pareceres sobre matérias relativas ao ensino superior, a serem submetidos, quando pertinentes, ao CNE. Entre tais matérias, estão a verificação de cursos (autorização, reconhecimento e renovação do reconhecimento); a definição de padrões de qualidade do ensino; os requisitos mínimos para o funcionamento dos cursos e o programa de avaliação dos mesmos. Para tanto a SESu conta com a assessoria e o apoio de Comissões de Especialistas de Ensino, por área de conhecimento.

A Comissão de Especialista de Ensino de Enfermagem (CEE-ENF), instituída por Portaria MEC/SESu n 076/96 (10/05/96), foi convocada pela Coordenação de Comissão de Especialistas de Ensino (COESP-SESu) para sua instalação e primeira reunião, no período de 10 a 12/06/96.

Atendendo à prioridade apresentada por essa Coordenação, a CEE-ENF elaborou a presente "descrição da área de enfermagem: oferta/demanda dos cursos de graduação" .

A superficialidade da descrição deve-se à precariedade de dados e indicadores a que se teve acesso, somada à exigüidade do tempo que se dispunha nessa primeira reunião.

\footnotetext{
' Documentário encaminhado pela Comissão de Especialistas de Ensino de Enfermagem à SESu/MEC, em 9 de junho de 1996

2 Prof essora Adjunta do Departamento de Enfermagem Materno - Infantil e de Saúde Pública da Escola de Enfermagem da UFMG. Coordenadora da Comissão de Especialistas de Ensino de Enfermagem SESu / MEC

${ }^{3}$ Professora do Departamento de Enfermagem da UNB. Membro da Comissão de Especialistas de Ensino de Enfermagem SESu /MEC

${ }^{4}$ Professora Adjunta do Departamento de Enfermagem Comunitária da Escola de Enfermagem da UFBA. Membro da Comissão da Especialistas de Ensino de Enfermagem SESu /MEC

${ }^{5}$ Professora Assistente do Departamento de Enfermagem de Saúde Pública da Faculdade de Enfermagem da UERJ. Membro da Comissão de Especialistas de Ensino de Enfermagem SESu/MEC.
} 
Acredita-se, no entanto, que através desta descrição como elaborada, é possível uma configuração geral da graduação em enfermagem, de forma a orientar os estudos e os trabalhos que serão realizados posteriormente, como por exemplo: a definição de padrões mínimos de qualidade e funcionamento dos cursos e a elaboração de um programa de avaliação dos mesmos, entre outros.

\section{INTRODUÇÃO}

Em 1993, o Ministério da Saúde - através da Coordenação Geral de Desenvolvimento de Recursos Humanos - CGRH/SUS- publicou, entre outros, o v. 2 da série "Cadernos RH/SAÚDE", dedicado especialmente à "Formação Superior em Saúde: tendências da graduação no período 1985/1991". As informações e indicadores apresentados têm como fonte o IBGE e o Ministério da Educação e do Desporto/Coordenação de Planejamento Setorial.

Se por um lado, a formação de profissionais de saúde é considerada como um dos eixos críticos e nucleares para a organização e eficiência do sistema de saúde, por outro, a situação e o movimento do setor de educação e as condições sob as quais a formação se processa, são elementos- chave para a definição de diretrizes e estratégias que orientem as políticas de formação de profissionais da área, na perspectiva do atendimento e cobertura de demandas e necessidades de saúde no País.

Nessa ótica, é que estudos referentes a qualquer curso, mesmo que simplesmente descritivos, não devem prescindir da relação e comparação entre o curso estudado e os demais cursos da área.

Considerando as informações e indicadores aglutinados pelo MS/CGRH/SUS, a presente descrição da área de enfermagem foi precedida de um perfil bastante genérico de alguns cursos de área de saúde (medicina, odontologia, farmácia, nutrição e fisioterapia), buscando, na medida do possivel, relacioná-los com a situação dos cursos de enfermagem e considerando o período 1985/1991. Através de indicadores que caracterizam a formação na área da saúde, é possível identificar a especificidade de determinado curso, na dinâmica da formação de profissionais do nível superior, na área da saúde. Caso contrário - tomar apenas um curso - tem-se visão estática e desarticulada da situação como um todo, e do curso estudado.

É preciso registrar, no entanto, a insuficiência e a imprecisão das informações e dados utilizados neste trabalho. Isto compromete, mas não anula, a possibilidade, neste primeiro momento, de considerá-los para o que pretende: uma descrição geral dos cursos de graduação na área de enfermagem, tendo como elementos descritores um conjunto de indicadores (demográficos, sócio econômicos e educacionais) estabelecidos pelo MEC/SESu. Ressalte-se, porém, que mesmo numericamente reduzidos, nem todos os descritores foram acessiveis no tempo que a CEE-ENF dispôs para elaborar este documento. 
CONSIDERAÇÕES GERAIS SOBRE A GRADUAÇÃO NA ÁREA DE SAÚDE: O CURSO DE ENFERMAGEM NA DINÂMICA DO PROCESSO DE FORMAÇÃO EM SAÚDE.

Pretendendo conferir alguma dinâmica à descrição sobre a graduação em enfermagem - objetivo precípuo deste documento - optou-se por situá-lo no movimento dos cursos da área da saúde. Para este fim, os cursos que estão considerados são apenas: medicina, nutrição, odontologia, fisioterapia e farmácia. A escolha destes cursos se deve aos dados que se teve acesso, através da citada publicação do Ministério da Saúde.

Considerando os cursos nomeados, havia no Pais, em 1990, 393 cursos de graduação (81 de odontologia; 80 de medicina; 47 de farmácia; 44 de fisioterapia; 39 de nutrição e 102 de enfermagem). A Região Sudeste e a Região Norte apresentavam, respectivamente, a maior e a menor concentração de cursos em todas estas carreiras ( BRASIL, 1993b).

Considerando dados do MEC/SESU/DAIN, houve um aumento de $9,4 \%$ no número de cursos nessa área, totalizando 430 cursos em 1994, sem contudo apresentar qualquer alteração na concentração regional, (TABELA 1).

TABELA 1 - NÚMERO DE CURSOS DE GRADUAÇÃO DA ÁREA DE SAÚDE E DISTRIBUIÇÃO PERCENTUAL POR REGIÃO GEOPOLITICA CEE-ENF BRASÍLIA, 1996

\begin{tabular}{|c|c|c|c|c|c|c|c|c|c|c|c|c|c|c|}
\hline \multirow{3}{*}{ REGIÕES } & \multicolumn{12}{|c|}{ CURSOS } & & \\
\hline & \multicolumn{2}{|c|}{ ENFERM } & \multicolumn{2}{|c|}{ ODONT. } & \multicolumn{2}{|c|}{ MEDIC. } & \multicolumn{2}{|c|}{ FISIOT. } & \multicolumn{2}{|c|}{ FARM } & \multicolumn{2}{|c|}{ NUTR. } & \multicolumn{2}{|c|}{ TOTAL } \\
\hline & $\mathbf{N}^{\mathbf{2}}$ & $\%$ & № & $\%$ & N & $\%$ & No & $\%$ & $\mathbf{N}^{\mathbf{2}}$ & $\%$ & № & $\%$ & $\mathbf{N}^{\mathbf{2}}$ & $\%$ \\
\hline NORTE & 07 & 6,5 & 02 & 02,3 & 04 & 05,0 & 01 & 01,7 & 03 & 05,7 & 01 & 02,4 & 018 & 004 \\
\hline NORDESTE & 22 & 20,4 & 13 & 14,9 & 13 & 16,0 & 07 & 11,9 & 08 & 15,1 & 08 & 19,1 & 071 & 017 \\
\hline SUDASTE & 51 & 47,2 & 50 & 57,5 & 44 & 54,3 & 35 & 59,3 & 25 & 47,2 & 22 & 52,4 & 227 & 053 \\
\hline SUL & 23 & 21,3 & 16 & 18,4 & 16 & 19,7 & 12 & 20,3 & 13 & 24,5 & 08 & 19,0 & 088 & 020 \\
\hline CENIRO-OASTE & 05 & 4,6 & 06 & 06,9 & 04 & 05,0 & 04 & 06,8 & 04 & 07,5 & 03 & 07,1 & 026 & 006 \\
\hline TOTAL & 108 & 25,0 & 87 & 19,0 & 81 & 20,0 & 59 & 14,0 & 53 & 12,0 & 42 & 10,0 & 430 & 100 \\
\hline
\end{tabular}

FONTE: MEC/SESU/DAIN, 1994a

Ainda sobre os cursos contemplados na tabela anterior, vale registro sobre: o número de diplomados, alunado, número de vagas oferecidas no vestibular, número de candidatos que se inscreveram às vagas oferecidas e afastamento do alunado dos cursos, no periodo considerado. 


\section{a) Sobre diplomados}

Conforme as informações estatísticas da CGRH/SUS (Brasil, 1993b), o número de diplomados nos cursos indicados da área da saúde diminuiu no período $1985-1990$ em todos os cursos $(1985=22.372$ graduados e $1990=$ 20.989). Esta tendência foi constatada em todas as regiões, sendo que o maior decréscimo ocorreu na Região Norte (-26\%), e o menor na Região e Sudeste $(-2 \%)$, e não se mostrou uniforme nos cursos, a saber:

- Medicina, Enfermagem e Nutrição apresentaram uma evolução negativa do número de diplomados, enquanto que Odontologia, Farmácia e Fisioterapia mostraram crescimento positivo;

- nos Cursos de Medicina e Enfermagem a evolução do crescimento no número de diplomados se manteve negativa em todo o período;

- apenas o Curso de Odontologia manteve a evolução positiva em todo o período referido;

- o decréscimo do número de graduados no periodo 1985/1990 ocorreu apenas nas instituições sob administração pública.

\section{b) Alunado}

Considerando o período em foco, o crescimento do alunado manteve-se constante, exceto no curso de enfermagem, que apresentou decréscimo no número de alunos a partir de 1988. Chama atenção que o decréscimo que se observa no período tenha ocorrido no setor público. No setor privado, observouse, para o mesmo período, um crescimento de $39 \%$ do corpo discente.

\section{c) Número de vagas oferecidas no vestibular}

Quanto ao número de vagas oferecidas nos vestibulares para estes cursos, constatou-se crescimento no período, no País como um todo e nas diversas Regiões Geo-Políticas. O maior crescimento foi nas Regiões Centro-Oeste $(65 \%)$ e Norte $(37 \%)$. Nas demais regiões, este crescimento variou em média $19 \%$. A evolução positiva no número de vagas/ vestibular foi mais ou menos uniforme em todos os cursos referidos, exceto no curso de Medicina, em que houve uma pequena retração.

\section{d) Procura pelos cursos}

Observa-se que a procura por esses cursos teve uma evolução positiva em todas as regiões do País, sendo que a maior proporção ocorreu nas Regiões Sul $(79 \%)$ e Centro-Oeste $(63 \%)$. Constatou-se também que $78 \%$ dos candidatos aos cursos da área de saúde procuram os cursos de Medicina (46\%) e de Odontologia (32\%).

As vagas oferecidas nesses cursos, no País, não foram totalmente preenchidas no período, sendo que dois cursos apresentaram preenchimento de vagas, predominantemente abaixo de 100\%: Enfermagem e Nutrição. 


\section{e) Afastamento do alunado dos cursos}

Um fenômeno é flagrantemente observável no período, em relação aos cursos em pauta: 0 afastamento dos alunos foi crescente e em proporções elevadas. Ocorreu em grau semelhante em todos os cursos e em todas as regiões. As maiores proporções ocorreram nos cursos de Enfermagem e Nutrição.

\section{f) Desempenho dos cursos}

Para considerar este aspecto, a referência utilizada foi a relação entre "matriculados na série inicial de cada curso" e o "número de diplomados no ano esperado". A base para estabelecer o "ano esperado" foi a duração mínima de cada curso como definido nos diplomas legais que dispõem sobre "currículo mínimo". Como não se dispunha de dados em série que permitissem considerar a duração mínima do curso de Medicina, tal curso não foi incluído neste grupo . Reconhece-se os limites desta relação e as múltiplas variáveis que interferem no desempenho de um curso. No entanto, sua presença nesta descrição é tão somente uma "amostra global" deste desempenho, objetivando alguma aproximação com esta questão na dinâmica da formação na área de saúde (TABELA 2).

\section{TABELA 2 - NÚMERO E RELAÇÃO DE MATRICULADOS NA SÉRIE INICIAL DIPLOMADOS NOS CURSOS, POR REGIÃO - CEE-ENF, BRASÍLIA, 1996}

\begin{tabular}{l|r|r|r|r|r|r|}
\hline \multicolumn{1}{|c|}{ ELEMENTOS / CURSOS } & \multicolumn{5}{c|}{ REGIOES } & \\
\hline & NORTE & NORD. & SUD. & \multicolumn{1}{c|}{ SUL } & C.OESTE & \multicolumn{1}{c|}{ BRASIL } \\
\hline 1-MATRICULA/SÉRIE INICIAL ENF. (1987) & 222 & 1394 & 3224 & 1405 & 239 & 6484 \\
\hline FAR. (1987) & 92 & 568 & 1964 & 940 & 90 & 3654 \\
\hline FISIOT. (1986) & 30 & 238 & 1451 & 419 & - & 2138 \\
\hline NUT. (1987) & 45 & 305 & 1420 & 506 & 117 & 2393 \\
\hline ODONT. (1986) & 119 & 744 & 4188 & 814 & 182 & 6047 \\
\hline TOTAL & 508 & 3249 & 12247 & 4084 & 628 & 20716 \\
\hline 2-DIPLOMADOS (1990) ENF. & 169 & 190 & 1549 & 590 & 132 & 3245 \\
\hline FAR. & 81 & 397 & 1295 & 610 & 69 & 2452 \\
\hline FISIOT. & 13 & 193 & 1193 & 347 & - & 1746 \\
\hline NUT. & 24 & 197 & 736 & 224 & 46 & 1227 \\
\hline ODONT. & 77 & 631 & 3800 & 736 & 117 & 5361 \\
\hline TO TAL & 379 & 2208 & 8573 & 2507 & 364 & 14031 \\
\hline 3-TAXA DE DIPLOMADOS (\%) ENF. & 76,126 & 56,67 & 48,04 & 41,99 & 55,23 & 50,04 \\
\hline FAR. & 88,04 & 69,89 & 65,93 & 64,89 & 76,76 & 67,10 \\
\hline FISIOT. & 43,33 & 81,09 & 82,21 & 82,81 & - & 81,66 \\
\hline NUT. & 53,33 & 64,59 & 51,83 & 44,26 & 39,31 & 51,27 \\
ODONT. & 64,70 & 84,81 & 90,73 & 90,41 & 64,28 & 88,65 \\
\hline
\end{tabular}

FONTE: BRASIL-MS/CGRH/SUS, 1993b

Estas informações referentes ao "movimento" de alguns cursos da área de saúde permitem um leque de hipóteses e apontam aspectos importantes a 
serem analisados. Isto significa, na prática, que a descrição da área como um todo, ou de apenas um determinado curso, é uma etapa que deve ser seguida de estudos mais específicos e sistematizados.

Entre as questões a serem melhor investigadas, objetivando uma análise da graduação na área de saúde estão:

- mercado de trabalho (emprego, salários), condições de trabalho);

- fatores sócio-culturais que conformam a expressão de profissão e do trabalho em saúde na sociedade.

Apenas como exemplo, os dados seguintes sobre empregos de saúde, segundo o IBGE/AMS (BRASIL,1993), apesar do tratamento sumário com que estão sendo apresentados, dão uma idéia dos seu possivel impacto na formação da área de saúde e, por via de conseqüência, na composição tecnológica da força de trabalho em saúde. $\quad \mathrm{Na}$ tabela 3 estão indicados apenas o emprego de profissionais de nivel superior e, entre estes, somente aqueles cujos cursos foram referidos neste documento.

TABELA 3 - EMPREgo DE SAÚDE DE DETERMINADOS PROFISSIONAIS DE SAÚDE, POR REGIÃO, CLASSIFICAÇÃO DO ESTABELECIMENTO, SEGUNDO IBGEIAMS - 1992, BRASIL, 1993 (1). CEE-ENF, BRASILIA, 1996 CEE-ENF, BRASILIAA, 1996.

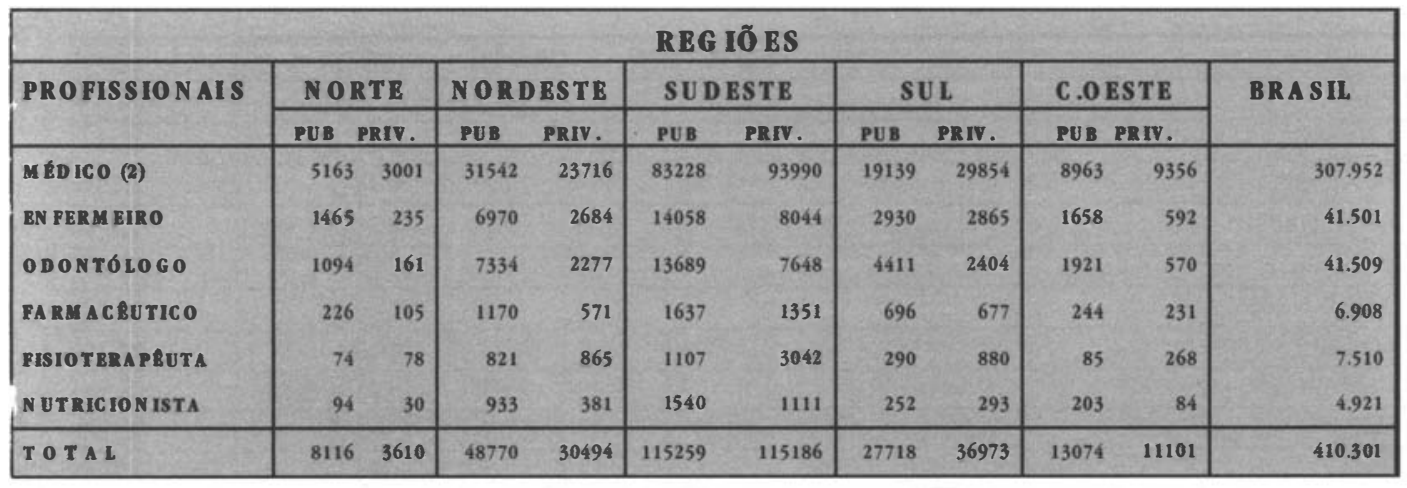

FONTE: IBGE/AMS (1992) . IN: BRASIL/MS/CGRH/SUS, 1993b

(1) os empregos referem-se aos postos de trabalho nos estabelecimentos cadastrados pela pesquisa

(2) nestes dados estão incluídos: Pediatra, Clínico Geral, Gineco-obstetra, Cirurgião, demais Esp. e Médicos Residentes.

A exemplo dos demais dados e informações estatísticas, estes sobre "emprego" pecam também pela fragilidade, por reduzirem por demais a questão do emprego na área de saúde. Vale o registro apenas como indicativo das possiveis relações entre a situação do mercado de trabalho/emprego e a formação na área de saúde. 
As observações seguintes, referentes a SALÁRIO ,têm como fonte o "GUIA DO ESTUDANTE - 94"- publicação da Editora Abril - disponivel nas bancas de revistas a cada dois anos. Seguramente há dados e estudos sobre emprego/salários consagrados pela alta qualidade. No entanto a escolha pelos dados pelo "Guia" deveu-se:

- primeiro, porque os dados e estudos não estavam acessíveis no tempo que se dispunha para elaborar esta descrição.

- segundo, porque o "Guia" tem como cliente preferencial os possíveis candidatos aos cursos da Universidade; logo suas informações estão ao alcance do grande público, o que não ocorre com estudos mais elaborados.

Independentemente do mérito que pode ser aferido à fonte destes dados sobre "salário", o importante a ser considerado é que eles, assim como os muitos outros contidos no "Guia", são os que estão acessíveis ao público em geral. Segundo esclarece a Editora da referida publicação, os dados e informações são resultantes de entrevistas feitas com: profissionais e entidades das diversas áreas; empresas de vários Estados; e, informações coletadas em escolas através de questionários. Feitas estas considerações, os salários são os seguintes:

- para o médico - a variação é de 2,5 SM (na Bahia) até 22,5 SM (Brasilia), sendo que neste "intervalo" encontram-se valores salariais os mais diversos;

- para o enfermeiro - variação entre 2,0 SM (Rio Grande do Sul) até 9,0 SM (Brasilia);

- para o odontólogo - variação entre 4,0 SM (Rio Grande do Sul) a 50 SM (Rio - Zona Sul/Consultório particular);

- para o nutricionista - variação entre 5 SM a 18 SM;

- para o farmacêutico - variação de 2,0 SM (Ceará, por 10 horas/semanais) a 30,0 SM (chefia em indústria)

- para o fisioterapêuta - variação entre 4,0 SM (RGS) e 15,0 SM.

Certamente esta visão panorâmica da formação na área de saúde, considerando alguns cursos e, entre eles, o Curso de Enfermagem, pretendeu tão somente situar a descrição específica subseqüente, na dinâmica do processo de formação profissional da área de saúde.

É necessário reiterar o caráter pontual da relação apresentada. No entanto, mesmo reconhecendo apriorísticamente seus limites, acredita-se que a descrição particular e isolada de apenas um curso da área comprometeria ainda mais a aproximação com a realidade. 


\section{A GRADUAÇÃO EM ENFERMAGEM: OFERTA/DEMANDA EVOLUÇÃO E TENDÊNCIAS}

Em outubro de 1972, na III Reunião Especial de Ministros da Saúde das Américas foi estabelecido o "Plano Decenal de Saúde para as Américas" (ORGANIZACION PANAMERICANA DE LA SALUD,1993), em cujas metas para 1980 foram incluidas algumas relativas ao desenvolvimento de recursos humanos para área de saúde. Tomar esta referência, transcorridas duas décadas e meia da sua definição, não é de todo improcedente, pelo menos no que diz respeito à área de enfermagem. Segundo o "Plano", a meta para a enfermagem/1980 seria:

- alcançar uma média de 4,5 enfermeiros/10.000 habitantes (ou seja 1 enfermeiro/2222 hab. aproximadamente) e 14,5 auxiliares de enfermagem/10.000 habitantes.

Considerando este propósito, buscou-se caracterizar a situação de enfermagem quanto ao número de enfermeiros (Tabela 4). Constata-se que em apenas 07 (sete) estados brasileiros a relação enfermeiro/habitante aproxima-se da meta prevista para 1980: Acre, Rio Grande do Norte, Paraíba, São Paulo, Rio de Janeiro, Rio Grande do Sul. Brasília.

TABELA 4 - RELAÇÃo ENTRE POPULAÇÃo GERAL do PAís, NÚMERO DE ENFERMEIROS INSCRITOS NO COFEN. BRASIL, 1996

\begin{tabular}{|c|c|c|c|}
\hline REGIĀO/ESTADO & $\begin{array}{l}\text { POPULACAO } \\
\text { RESIDENIE (EST. } \\
\text { 1994) (1) } \\
\end{array}$ & $\begin{array}{l}\text { ENFERMERROS } \\
\text { INSCRIOS NO } \\
\text { COFEN (UUL95) (2) } \\
\end{array}$ & $\begin{array}{l}\text { RELAC̄̄O } \\
\text { ENF/HABITANTE } \\
\end{array}$ \\
\hline $\begin{array}{l}\text { NORTE } \\
\text { Amazonas } \\
\text { Pará } \\
\text { Acre } \\
\text { Amapá } \\
\text { Rondônia } \\
\text { Roraima } \\
\text { Tocantins } \\
\end{array}$ & $\begin{array}{r}11.456 .166 \\
2.316 .000 \\
5.673 .083 \\
456.253 \\
331.311 \\
1.419 .005 \\
283.669 \\
976.865 \\
\end{array}$ & $\begin{array}{r}2.934 \\
564 \\
1.598 \\
186 \\
74 \\
334 \\
53 \\
53 \\
125 \\
\end{array}$ & $\begin{array}{l}1 / 3.905 \\
1 / 4.106 \\
1 / 3.550 \\
1 / 2.453 \\
1 / 4.477 \\
1 / 4.249 \\
1 / 5.352 \\
1 / 7.815 \\
\end{array}$ \\
\hline $\begin{array}{l}\text { NOROESTE } \\
\text { Maranhāo } \\
\text { Piauí } \\
\text { Ceará } \\
\text { Rio Grande do Norte } \\
\text { Paraiba } \\
\text { Pernambuco } \\
\text { Nlagoas } \\
\text { Sergipe } \\
\text { Bahia } \\
\end{array}$ & $\begin{array}{l}44.735 .163 \\
5.210 .194 \\
2.716 .732 \\
6.679 .381 \\
2.577 .051 \\
3.329 .212 \\
7.389 .616 \\
2.680 .114 \\
1.606 .114 \\
1.253 .673 \\
\end{array}$ & $\begin{array}{r}13.772 \\
664 \\
559 \\
2.589 \\
1.253 \\
1.952 \\
2.060 \\
637 \\
491 \\
3.567 \\
\end{array}$ & $\begin{array}{l}1 / 3.248 \\
1 / 7.847 \\
1 / 4.860 \\
1 / 2.560 \\
1 / 2.057 \\
1 / 1.705 \\
1 / 3.591 \\
1 / 4.207 \\
1 / 3.271 \\
1 / 3.515 \\
\end{array}$ \\
\hline \begin{tabular}{|l} 
SUDESTE \\
Espirito Santo \\
Minas Gerais \\
Rio de Janeiro \\
Săo Paulo \\
\end{tabular} & $\begin{array}{r}65.323 .825 \\
2.781 .624 \\
16.461 .740 \\
12.961 .740 \\
33.118 .828 \\
\end{array}$ & $\begin{array}{r}32.197 \\
553 \\
4.311 \\
9.462 \\
17.871 \\
\end{array}$ & $\begin{array}{l}1 / 2.029 \\
1 / 5.030 \\
1 / 3.818 \\
1 / 1.370 \\
1 / 1.853 \\
\end{array}$ \\
\hline \begin{tabular}{|l} 
SUL \\
Paraná \\
Santa Catarina \\
Rio Grande do Sul \\
\end{tabular} & $\begin{array}{r}23.001 .473 \\
8.643 .822 \\
4.821 .522 \\
9.536 .129 \\
\end{array}$ & $\begin{array}{l}9.939 \\
2.757 \\
1.736 \\
5.446 \\
\end{array}$ & $\begin{array}{l}1 / 2.314 \\
1 / 3.135 \\
1 / 2.777 \\
1 / 11.751 \\
\end{array}$ \\
\hline $\begin{array}{l}\text { CENTRO-OESTE } \\
\text { Distrito Federal } \\
\text { Goils } \\
\text { Mato Grosso } \\
\text { Mato Grosso do Sul } \\
\end{array}$ & $\begin{array}{r}10.320 .777 \\
1.734 .620 \\
4.313 .632 \\
1.909 .847 \\
2.362 .676 \\
\end{array}$ & $\begin{array}{r}3.588 \\
1.728 \\
1.044 \\
59 \\
267 \\
\end{array}$ & $\begin{array}{l}1 / 2.876 \\
1 / 1.004 \\
1 / 4.132 \\
1 / 3.610 \\
1 / 8.232 \\
\end{array}$ \\
\hline BRASIL & 154.837 .424 & 62.430 & $1 / 2.477$ \\
\hline
\end{tabular}

FONTES : (1) IBGE_Projeção para junho de 1994. IN: BRASIL, MEC/SESu/DAIN,1994b. (2) COFEN_ Inscritos até 31.07.1995 
Esta situação não permite nenhuma afirmação quanto à sua correspondência com quaisquer indicadores, sejam econômicos, políticos ou sociais. Por hipótese, simplesmente ocorreu à revelia de qualquer estratégia ou intervenção formal.

Para 1980, este déficit de enfermeiros já estava previsto, como pode ser constatado nos ANAIS DA $V$ CONFERÊNCIA NACIONAL DE SAÚDE (BRASIL,1993a ).

"Quanto ao ensino superior, a comportamento atual do sistema garante até 1980, a atendimento das necessidades profissionais em Medicina, Odontologia e Farmácia, tomando-se por base as metas recomendadas pelo Plano Decenal de Saude para as Américs.... O mesmo não ocorre quanto à enfermagem, em velação à qual se node constatar acentuado déficit de profissionais. Tomando-se ainda por base a recomendação do Plano Decenal de Saúde para as Américas, teriamos em 1980, se marstido a atual ritmo de formação, um déficit de $\mathbf{3 8 . 6 0 0}$ enfermeiros "grifo nosso).

Segundo o Prof. Edson Machado de Souza, conferencista dessa V CNS, com o tema "Política de Formação de Recursos Humanos para o Setor Saúde", este déficit de enfermeiros seria corrigido graças à providências que estavam sendo tomadas pelo MEC "não apenas para fomentar o aumento de vagas nos atuais cursos de enfermagem, como também para criar novos cursos, junto às instituições federais e em regiões ainda não servidas" (BRASIL, 1993a ).

Realmente, houve crescimento significativo no número de cursos e vagas de enfermagem a partir de 1976. No entanto, a situação atual demonstra um déficit em torno de 10.000 enfermeiros, caso a situação de saúde do País e as demandas dos serviços de saúde estivessem estacionadas em 1980. Logo, não temos apenas um déficit somado a dezesseis anos de atraso. Cabe ressaltar que o número de enfermeiros considerado na Tabela 4, refere-se aos que estão inscritos no COFEN/COREN's, e não àqueles que estão efetivamente inseridos no setç de produção de assistência à saúde. Caso fossem considerados apenas os que estão em exercício no setor de produção de serviços assistenciais de saúde, este déficit seria maior .

Um fator que seguramente interfere no déficit de enfermeiros refere-se à geração de empregos. No estudo realizado por Nogueira (1986) sobre "A Dinâmica do Mercado de Trabalho em Saúde no Brasil 1970-1983", constata-se que de 1977 à 1983 foram criados 7.629 empregos para 23.033 enfermeiros formados no período. Isto significa que a criação de novos e o aumento de vagas nos cursos de graduação em enfermagem, isolada e desarticulada de medidas e intervenções no mercado de trabalho, no processo de formação, e nas condições de trabalho da enfermagem e da saúde, poderão, inclusive, gerar efeito contrário: aumenta-se o número de diplomados e de desempregados. De 
1975 a 1977 foram implantados 22 novos cursos de enfermagem em todo o País. Em 1980 o número de cursos totaliza 79 e, atualmente, temos 108 cursos. Outro aspecto/conseqüência a ser considerado é que 0 aumento de cursos/vagas, concomitantemente com a reduzida criação de empregos, poderá interferir na procura pelo curso e no afastamento do aluno. O estudo do Ministério da Saúde/Coordenadoria Geral de Desenvolvimento de Recursos Humanos/SUS ( BRASIL,1993b) para o período de 1985-1991, é importante para conformar o perfil dos cursos em relação à número de inscritos no Vestibular, matrículas na série inicial dos cursos e número de alunos afastados. Segundo a análise realizada pelo MS/CGRH/SUS vale ressaltar que:

- "a graduação de enfermeiros vem sofrendo uma diminuição crescente, tendência observada e todas as regiões. $O$ déficit do número de diplomados é o maior entre as carreiras de saúde.

- apesar do crescimento, a oferta e a procura dos cursos de Enfermagem, são as menores, entre as carreiras de saúde.

- o afastamento dos cursos de Enfermagem, alto no período, é o maior, no conjunto das carreiras de saúde". (grifo nosso).

TABELA 5 - NÚMERO DE INSCRIÇÕES NO VESTIBULAR, DE MATRICULADOS NA SÉRIE INICIAL E DE AFASTAMENTOS DOS CURSOS DE GRADUAÇÃO ENFERMAGEM, 1986/1991.CEE-ENF, BRASÍLIA, 1996

\begin{tabular}{|c|c|c|c|}
\hline \multirow{2}{*}{ ANO } & \multicolumn{3}{|c|}{ SITUAÇÕES } \\
\cline { 2 - 4 } & No MAT. VEST. & MAT. NA SÉRIE INICIAL & No AFASTAMENTOS \\
\hline 1986 & 23.633 & 5553 & 1446 \\
1987 & 25.956 & 6484 & 1649 \\
1988 & 23.686 & 5980 & 3453 \\
1989 & 21.856 & 7244 & 3379 \\
1990 & 24.432 & 6108 & 3792 \\
1991 & 28.192 & 6247 & 3592 \\
\hline
\end{tabular}

FONTE:BRASIL- MS/CGRH/SUS,1993b.

No mesmo período, cresceu a oferta de vagas no país $(10 \%)$, com incremento máximo de $90 \%$ na região Norte, e mínimo (1\%) na região Nordeste. A procura aos cursos aumentou em $10 \%$ no país, com $162 \%$ de incremento na região Centro-Oeste. Nas regiões Nordeste e Sudeste o incremento foi apenas de $5 \%$ e $8 \%$,respectivamente. Apesar do incremento, os cursos de enfermagem são os menos procurados na área da saúde e os que apresentaram a maior proporção de afastamentos no período ( $148 \%)$; o incremento de afastamento foi maior na região Nordeste (324\%) e menor na região Sul $(91 \%)$. 
A ausência de dados para os anos de 1992, 1993, 1994 e 1995 não permitiu a avaliação da evolução destas tendências para este período. Os dados disponiveis para 1994 permitem apenas uma observação pontual, o que compromete qualquer análise de tendência.

Anteriormente fez-se a relação enfermeiro/habitante por Região, constatando-se um déficit significativo, mesmo tomando como referência a meta pretendida para 1980. Se por um lado o incremento no número de diplomados depende, entre outros, de fatores relativos ao mercado de trabalho, e não propriamente referente ao processo de formação, por outro lado, conhecer as possiveis variáveis que conformam a maior ou menor procura e ingresso aos cursos torna-se necessário. Para tanto seria imprescindivel um estudo centrado na população potencial de "clientes" dos cursos superiores, em especial aqueles que procuram cursos da área de saúde/enfermagem. Em tese, jovens na faixa etária de 18-24 anos, concluintes do II grau, seriam o objeto deste estudo. Em que pese o acesso ao número de concluintes do II Grau para 1991/1992/1993, e ao número de jovens na faixa etária citada (para 1991), tais dados foram insuficientes para quaisquer projeções quanto ao comportamento da possivel clientela aos cursos da área de saúde/enfermagem.

Um aspecto que merece destaque é o crescimento do setor privado no oferecimento de cursos/vagas no ensino superior no País (TABELA 6).

\section{TABELA 6 - DISTRIBUIÇÃO DO NÚMERO DE CURSOS DE SAÚDE SEGUNDO CATEGORIA PROFISSIONAIS E DEPENDÊNCIA ADMINISTRATIVA DAS INSTITUIÇÕES MANTENEDORAS. BRASIL, 1990}

\begin{tabular}{l|c|c|c|c|c|c}
\multirow{2}{*}{ CURSOS } & \multicolumn{4}{|c|}{ PÚBLICA } & PRIVADA & TOTAL \\
\cline { 2 - 5 } & FEDERAL & ESTADUAL & MUNICIPAL & TOTAL & & \\
\hline MEDICINA & 32 & 10 & 03 & 45 & 35 & 80 \\
ODONTOLOGIA & 27 & 15 & 04 & 46 & 35 & 81 \\
ENFERMAGEM & 33 & 19 & 05 & 57 & 45 & 102 \\
FARMÁCIA & 20 & 08 & 01 & 29 & 18 & 47 \\
NUTRIÇĀO & 18 & 04 & - & 22 & 17 & 39 \\
FISIOTERAPIA & 06 & 05 & 02 & 13 & 31 & 44 \\
TOTAL & 136 & 61 & 15 & 212 & 181 & 393 \\
\hline
\end{tabular}

FONTE: BRASIL- MS/CGRH/SUS, 1993b

Quanto aos Cursos de Enfermagem, o trabalho de Fernandes (1994) analisa a retração do setor público e o avanço do setor privado, apresentando os seguintes dados para confirmar este processo (Tabelas 7 e 8). 
TABELA 7 - CRESCIMENTO DOS CURSOS DE GRADUAÇÃO EM ENFERMAGEM POR DEPENDÊNCIA ADMINISTRATIVA. BRASIL 1965 A 1993

\begin{tabular}{|l|c|c|c|c|c|c|}
\hline $\begin{array}{l}\text { DEPENDENCIA } \\
\text { ADMINISTRATIVA }\end{array}$ & $\begin{array}{l}\text { CURSOS } \\
\text { EXISTENTES } \\
\text { EM } 1965\end{array}$ & \multicolumn{2}{l|}{$\begin{array}{l}\text { CURSOS IMPLANTADOS } \\
\text { ENTRE 1966 E 1993 }\end{array}$} & \multicolumn{2}{|c|}{ TOTAL } \\
\cline { 2 - 7 } & F & $\%$ & F & $\%$ & F & $\%$ \\
\hline PÚBLICO & 12 & 46,2 & 22 & 27,5 & 34 & 32,1 \\
- FEDERAL & 06 & 23,1 & 11 & 13,8 & 17 & 16,1 \\
- ESTADUAL & 01 & 03,8 & 07 & 08,7 & 08 & 07,5 \\
\hline PARTICULAR & 07 & 26,9 & 40 & 50,0 & 47 & 44,3 \\
\hline TOTAL & 26 & 100,0 & 80 & 100,0 & 106 & 100,0 \\
\hline
\end{tabular}

FONTE: FERNANDES, J.D. - IN: REVISTA BRASILEIRA DE ENFERMAGEM v.47,n .2, p. 149, abr./jun. 1994

Outra constatação da autora é que o incremento do número de cursos de graduação em enfermagem por dependência administrativa no período 1965/93 foi de $210,5 \%$ no setor público e $571,4 \%$ no particular.

TABELA 8 - CURSOS DE GRADUAÇÃO EM ENFERMAGEM, POR REGIÃO GEOGRÁFICA E DEPENDÊNCIA ADMINISTRATIVA. BRASIL, 1993

\begin{tabular}{|c|c|c|c|c|c|c|c|c|}
\hline \multirow{3}{*}{$\begin{array}{l}\text { DEPENDÊNCIA } \\
\text { REGIŐES }\end{array}$} & \multicolumn{6}{|c|}{ PÚBLICO } & \multirow{2}{*}{\multicolumn{2}{|c|}{ PARTICULAR }} \\
\hline & \multicolumn{2}{|c|}{ FEDERAL } & \multicolumn{2}{|c|}{ ESTADUAL } & \multicolumn{2}{|c|}{ MUNICIPAL } & & \\
\hline & $\mathbf{F}$ & $\%$ & $\mathbf{F}$ & $\%$ & $\mathbf{F}$ & $\%$ & $\mathbf{F}$ & $\%$ \\
\hline NORTE & 05 & 14,7 & 01 & 05,9 & - & - & 01 & 02,1 \\
\hline NORDESTE & 09 & 26,5 & 07 & 41,2 & 01 & 12,5 & 05 & 10,6 \\
\hline CENTRO-OESTE & 04 & 11,8 & - & - & - & - & 01 & 02,1 \\
\hline SUDESTE & 10 & 29,4 & 06 & 35,3 & 04 & 50,0 & 30 & 63,9 \\
\hline SUL & 06 & 17,6 & 03 & 17,6 & 03 & 37,5 & 10 & 21,3 \\
\hline TOTAL & 34 & 100,0 & 17 & 100,0 & 08 & 100,0 & 47 & 100,0 \\
\hline
\end{tabular}

FONTE: FERNANDES, J.D. - IN: REVISTA BRASILEIRA DE ENFERMAGEM, v.47, n. 2, p. 149 abr.Jjun. 1994 
Finalizando, ainda algumas outras considerações sobre os Cursos de Enfermagem:

- a Portaria MEC n $1721 / 94$ define os mínimos de conteúdo e duração (mínimo de 4 e máximo de 6 anos) dos cursos de enfermagem. 0 tempo de adaptação a esta nova determinação é até 1997 (Portaria MEC 01/96, complementar ¿̇ 1721/94 ). Alguns pontos da Portaria $1721 / 94$ vêm sendo formalmente questionados pelas Escolas/Cursos de Enfermagem e Associação Brasileira de Enfermagem. Entre eles, a distribuição da carga horária por áreas do conteúdo, assim como a exclusão da licenciatura (conteúdos pedagógicos), vêm sendo reiteradamente apresentados como matérias a serem examinadas, na perspectiva da supressão da distribuição de carga horária/áreas e inclusão de conteúdos pedagógicos. Sobre este último aspecto, cabe registrar que uma atividade intrínseca do enfermeiro, prevista na lei do exercicio profissional, refere-se à sua participação na formação das habilitações de nivel médio (auxiliares e técnicos de enfermagem) .

- Em razão da total falta de acesso aos dados referentes a corpo docente das Escolas/Cursos de Graduação em Enfermagem, este aspecto de formação ficou ausente deste documento.

- Quanto à pós-graduação em enfermagem, os dados disponiveis, no momento, concentram-se no número dos cursos existentes (14 mestrados e 07 doutorados) ${ }^{10}$ que repetem a concentração regional observada na graduação. A pós-graduação "lato sensu" - considerada muito importante para a área assistencial e de gerência de serviços de enfermagem - também não está contemplada pelo mesmo motivo: falta de acesso aos dados possivelmente existentes.

\section{CONSIDERAÇÕES FINAIS}

A descrição da área de enfermagem aqui apresentada é a etapa inicial de um trabalho a ser desenvolvido pela CEE-ENF, objetivando assessorar o MEC/SESu na definição de politicas para a formação de enfermeiros. De conformidade com a demanda e urgência, expressas quando de instalação da Comissão, esta descrição se caracteriza pelo tratamento horizontal da situação, que na prática é muito complexa. No entanto, enquanto etapa preliminar, mesmo com este limite, poderá facilitar o encaminhamento dos trabalhos subseqüentes.

É oportuno registrar que a qualidade e eficiência ao trabalho da CEE-ENF dependem da infra-estrutura que facilite a operacionalização da produção da COMISSÃO.

A reativação das Comissões de Especialistas de Ensino no MEC é consensualmente avaliada como uma estratégia que poderá causar impacto 
positivo na educação de nível superior. No caso de enfermagem, tanto Escolas como Entidades já haviam se manifestado pelo retorno desta prática. Os atuais membros da CEE-ENF se dispõem ao cumprimento das suas atribuições, inclusive articulando-se com Escolas/Cursos e Entidades de Enfermagem, o que seguramente conferirá melhor desempenho à Comissão.

\section{REFERÊNCIAS BIBLIOGRÁFICAS}

1. BRASIL - Ministério da Saúde. Coordenação de Recursos Humañs/SUS. Cadernos RH Saúde. Brasília: Ministério da Saúde -CRH/SUS, 1993a, v.1, n.1.

2. Cadernos RH Saúde. Brasília: Ministério da Saúde-CRH/SUS, 1993 b v.1, n.2

3. BRASIL - Ministério da Educação e do Desporto. SESu / DAIN Cursos, alunado, inscrições vagas e ingressos, 1994 a (xerocópia ).

4. Ministério da Educação e do Desporto.SESu /DAIN. Projeção da População Brasileira para 1994 - IBGE . 1994 b (xerocópia).

5. BRASIL -CONSELHO FEDERAL DE ENFERMAGEM.. População de profissionais de enfermagem cadastrados até 35/7/95. Rio de Janeiro: COFEN, 1995.

6. GUIA DO ESTUDANTE 94. Tudo sobre profissões e cursos de nível superior do país. São Paulo: Editora Abril, 184p.

7. FERNANDES, Josicélia D. A privatização do ensino de enfermagem no Brasil: economia da qualidade. R. bras. Enferm., Brasília, v. 47, n.2, p 144-159, abr./jun. 1994.

8. NOGUEIRA, Roberto P. Dinâmica do Mercado de Trabalho em Saúde no Brasil:1970 -1983. Brasília: OPS, 1986, 55p.

9. ORGANIZACION PANAMERICANA DE LA SALUD. Plan Decenal de Salud para Las Americas. III Reunión de Ministros de Salud de las Américas. 1973, Santiago, Chile. Informe Final... Washington, D.C.:Organizacion Panamericana de la Salud, (Documento Oficial $n^{\circ}$. 118).

10. CAPES - Catálogo de Cursos de Mestrado e Doutorado 1993 referentes ao biênio 92/93. - Avaliação da Pós-Graduação - Síntese dos resultados / 1994. 